\title{
Cell secretome based approaches in Parkinson's disease regenerative medicine
}

\author{
Cláudia R. Marques ${ }^{\mathrm{a}, \mathrm{b}}$, Ana Marote ${ }^{\mathrm{a}, \mathrm{b}}$, Bárbara Mendes-Pinheiro ${ }^{\mathrm{a}, \mathrm{b}}$, Fábio G. Teixeira ${ }^{\mathrm{a}, \mathrm{b}}$ and António J. Salgado \\ aLife and Health Sciences Research Institute (ICVS), School of Medicine, University of Minho, Braga, Portugal; 'bICVS/3B's - PT Government Associate \\ Laboratory, Braga/Guimarães, Portugal
}

\section{ABSTRACT}

Introduction: The available therapeutic strategies for Parkinson's disease (PD) rely only on the amelioration of the symptomatology of the disease, lacking neuroprotection or neuroregeneration capacities. Therefore, the development of disease modifying strategies is extremely important for the management of PD in the long term.

Areas covered: In this review, the authors provide an overview of the current therapeutic approaches for PD and the emerging use of stem cell transplantation as an alternative. Particularly, the use of the secretome from mesenchymal stem cells (MSCs), as well as some methodologies used for the modulation of their paracrine signaling, will be discussed. Indeed, there is a growing body of literature highlighting the use of paracrine factors and vesicles secreted from different cell populations, for this purpose.

Expert opinion: Secretome from MSCs has shown its potential as a therapy for PD. Nevertheless, in the coming years, research should focus in several key aspects to enable the translation of this strategy from the bench to the bedside.
ARTICLE HISTORY

Received 3 May 2018 Accepted 7 November 2018

\section{KEYWORDS}

Biomaterials; bioreactors; dopamine; dopaminergic neurons; hypoxia; mesenchymal stem cells; neural derived cells; Parkinson's disease; secretome

\section{Introduction}

Parkinson's disease (PD) is a chronic neurodegenerative disorder of insidious onset, characterized by the presence of predominantly motor symptomatology such as bradykinesia, rest tremor, rigidity, and postural disturbances [1]. These motor deficits are the result of the progressive loss of dopaminergic (DAergic) neurons in the substantia nigra pars compacta $(\mathrm{SNpc}$ ) leading to the denervation of the nigrostriatal tract and significant reduction of dopamine (DA) at the striatal level $[2,3]$. Current theories on the etiology of PD consider this disorder to be multifactorial and the result of a genetic predisposition in combination with environmental factors. These phenomena are thought to be responsible for the cellular changes leading to progressive neuronal degeneration in which oxidative stress, mitochondrial dysfunction, and failure of the protein degradation machinery are probably involved. One of the aims of the current PD research is to elucidate the sequence in which they act and understand the interaction between these pathways to develop effective neuroprotective strategies for PD [4]. Indeed, the treatment for PD has not changed substantially in the past 50 years, with DA replacement therapy using levodopa (L-DOPA) as the mainstay [5]. However, its extended use, associated with the needs of increased dosages, is linked with secondary side effects such as nausea, vomit, hypotension, and long-term complications including motor fluctuations and dyskinesias [6]. New drugs in the form of DA agonists or enzyme inhibitors have been developed to delay the need of L-DOPA therapy or to reduce its dosage $[7,8]$. Moreover, surgical treatments, such as deep brain stimulation (DBS) in the globus pallidus internus, or in the subthalamic nucleus, have been applied as an alternative in patients with significant motor complications that no longer respond to pharmacological approaches [9]. However, these are all symptomatic approaches with no proven effect on disease progression, imposing the need for innovative therapeutic approaches.

Over three decades ago, several research groups have devoted to the development of cell-based dopamine replacement strategies [10]. While the capacity of human fetal ventral midbrain (fVM) grafts to induce clinical improvement when transplanted into the human brain was being achieved and evaluated, other cell sources including embryonic porcine ventral mesencephalic tissue, autologous carotid body cells or adrenal medullary tissue and human retinal pigmentary epithelial cells underwent clinical trials. However, these trials were unsuccessful, maybe as a result of limited pre-clinical data to support their use $[10,11]$. Regarding the use of fVM allografts, they proved to be effective just in certain patients. This inconsistency is justified by differences in tissue preparation, patient selection or also surgical method, among other factors [11]. However, these studies contributed to further knowledge that enabled the development of a new human fVM trial in PD, the TRANSEURO, funded in 2009 by the European Union. Although this strategy seems promising, it is not a realistic option for large-scale applications, in which a cell source that is easily stored, available and self-renewable is more advantageous. Therefore, stem cells emerged as an attractive alternative [10]. Among stem cell populations, the 


\section{Article highlights}

- The available therapeutic strategies for Parkinson's disease do not impact disease progression, imposing the need for innovative therapeutic approaches.

- The use of stem cells has emerged as a promising approach for regenerative medicine.

- Cell sources such as embryonic stem cells, induced pluripotent stem cells, neural stem cells, or mesenchymal stem cells are some of the most promising to replacement strategies.

- The secretome of some cell populations is now accepted as their main therapeutic action.

- The secretome can be modulated at intracellular and extracellular levels with the purpose to potentiate its benefits.

- The potential of the secretome in Parkinson's disease encourages the development of cell-free products over cell transplantation strategies.

This box summarizes key points contained in the article.

use of human embryonic stem cells (hESCs) (NCT019636), induced pluripotent stem cells (iPSCs), or neural stem cells (NSCs) (NCT02452723) to create DA neurons to restore the function of brain affected regions are some of the most promising cell sources to replacement strategies [12]. Other approaches have explored the use of non-ectodermal origin stem cells, such as mesenchymal stem cells (MSCs). Indeed, the use of these cells for regeneration purposes in different neurological disorders have been extensively studied [13]. However, the approach vary according to the disease and is completely different for PD and Huntington disease (HD) in comparison with stroke or traumatic brain injury (TBI) $[14,15]$. At the onset of PD and HD a considerable number of neurons are already lost. Therefore, a regenerative therapy in this context will potentially stimulate the differentiation of new cells for the replacement of the ones that are already dead. In the particular case of PD, a reactivation of cells that are still viable but dysfunctional or in a dormant phase might also happen [16]. On the other hand, a regenerative cell therapy for stroke or TBI is normally administered in an acute stage with the purpose of attenuating the inflammation process, which is a characteristic of this stage [15]. Therefore, in these diseases,

100 the regenerative therapy would have a more protective role upon the cells that are still viable.
Even though much effort has been applied to the development of cell therapies, we still lack a clinically competitive treatment for people with PD. Currently, according to the US National Institutes of Health website http://www.ClinicalTrials. gov, there are two active (not recruiting) clinical trials using cells in the context of this disease. Seven are recruiting (phases I or II), involving the use of MSCs, neural precursor cells derived from fetal mesencephalic or hESCs, human parthenogenetic neural stem cells, and bone marrow-derived stem cells. Regarding the finished studies, four were completed (Table 1), one was withdrawn (company was dissolved), other one was terminated due to difficulties in recruiting the adequate number of patients timely. Lastly, one study was completed but denoted as 'terminated' (Table 1).

For the application of the generated DAergic neurons in a clinical setting, pre-clinical trials should demonstrate that these cells: i) can survive in large numbers upon transplantation; ii) are able to effectively reinnervate the nigrostriatal tract; iii) have the capacity for axonal outgrowth and DA release; and iv) lead to behavioral improvements in animal models of PD [17]. Moreover, in such a disease in which its pathogenesis is still unclear, experimental work is mandatory to answer to specific questions such as the correlation of age and PD or which is the role of Lewy Bodies in the initiation or progression of the disease, in order to uncover molecular targets for innovative treatments. Therefore, investment in the generation of pre-clinical data should be pursued to develop clinical studies with stronger evidence of clinical benefits and demonstrated mechanisms of action.

A recent paradigm shift has emerged suggesting that the beneficial effects of stem cells may not be restricted to cell transplantation/differentiation alone but could be mediated by the secretion of bioactive molecules, which nowadays is referred as secretome [18]. This could be advantageous when compared to the cell-replacement strategies. Indeed these latter still present several drawbacks, namely the need of to obtain high number of cells to compensate for their low rate survival after transplantation, the possibility of eliciting an immune response if allogenic cells are used and the uncertain destiny of systemically administered cells [19-21].

Cells' secretome includes a broad repertoire of trophic factors, immunomodulatory cytokines, lipids, and extracellular

Table 1. Clinical approaches using potential cell therapies for PD regeneration. (PD = Parkinson's disease; DBS = deep brain stimulation).

\begin{tabular}{|c|c|c|c|c|}
\hline & Therapy & Aim of the study & Comments & $\begin{array}{l}\text { ClinicalTrials. } \\
\text { gov identifier }\end{array}$ \\
\hline \multirow[t]{2}{*}{$\begin{array}{l}\text { Active (not } \\
\text { recruiting) }\end{array}$} & $\begin{array}{l}\text { Stereotactic intraputaminal implantation of human } \\
\text { allogeneic fetal derived stem cells }\end{array}$ & $\begin{array}{l}\text { Safety trial pathology } \\
\text { of the disease }\end{array}$ & Phase I ongoing & NCT02780895 \\
\hline & $\begin{array}{l}\text { Xenotransplantation of immunoprotected (alginate- } \\
\text { encapsulated) porcine choroid plexus cells }\end{array}$ & $\begin{array}{l}\text { Safety and effect on } \\
\text { progression of PD }\end{array}$ & Phase II ongoing & NCT02683629 \\
\hline \multirow[t]{5}{*}{ Completed } & Embryonic dopamine cell implant surgery & $\begin{array}{l}\text { Effect on progression } \\
\text { of PD }\end{array}$ & $\begin{array}{l}\text { Phase III: clinical benefit and graft viability } \\
\text { sustained up to } 4 \text { years after transplantation }\end{array}$ & NCT00038116 \\
\hline & Transplantation of fetal porcine cells & $\begin{array}{l}\text { Safety and effect on } \\
\text { progression of PD }\end{array}$ & Phase II (no posted conclusions) & NCT00226460 \\
\hline & $\begin{array}{l}\text { Xenotransplantation of immunoprotected (alginate- } \\
\text { encapsulated) porcine choroid plexus cells }\end{array}$ & $\begin{array}{l}\text { Safety and effect on } \\
\text { progression of PD }\end{array}$ & Phases I/II (no posted conclusions) & NCT01734733 \\
\hline & $\begin{array}{l}\text { Implantation of peripheral nerve graft during DBS } \\
\text { surgery }\end{array}$ & $\begin{array}{l}\text { Safety data for a larger } \\
\text { phase clinical trial }\end{array}$ & $\begin{array}{l}\text { Pilot study: feasible and safe delivery of the } \\
\text { graft; possible clinical benefit }\end{array}$ & NCT01833364 \\
\hline & $\begin{array}{l}\text { Bilateral implantation of cultured human retinal } \\
\text { pigment epithelial cells on microcarriers }\end{array}$ & $\begin{array}{l}\text { Safety and effect on } \\
\text { progression of PD }\end{array}$ & $\begin{array}{l}\text { Phase II: no advantage over sham surgery for } \\
\text { advanced PD }\end{array}$ & NCT00206687* \\
\hline
\end{tabular}

\footnotetext{
* Completed study but registered as terminated, because lifelong extended follow-up phase was discontinued after 12 years.
} 
vesicles, which in turn have multiple implications in the regulation of key biologic processes such as neuroprotection (e.g. protecting cells from oxidative stress), neurodifferentiation, as well as regulation of inflammatory processes [22]. Here, we specifically highlight the current understanding regarding the use of NSCs, glial cells and MSCs as natural sources or as vehicles for the delivery of neurotrophic factors in the context of PD. Moreover, we review recent experimental data addressing different methodologies being used for an efficient and high-quality secretome production.

\section{Therapeutic strategies for PD based on cell secretome}

\subsection{Neural-derived cells}

Neural stem cells are the obvious candidates for cell transplantation strategies due to their ability to self-renew and differentiate into neurons. Besides the graft-derived functional effects when transplanted into the striatum of 6-hydroxydopamine (6-OHDA)-lesioned rats, Yasuhara and colleagues [23] further demonstrated the neuroprotective effects of these cells mediated by trophic factor secretion.

Recent work from our lab as shown that the injection of the secretome of human neural progenitor cells (hNPCs) in the $\mathrm{SNpc}$ and striatum of a 6-OHDA rat model of PD stimulated the recovery of DAergic neurons, resulting in an improvement of the motor behavior [24]. Moreover, the improvement in DAergic neurons survival and motor deficits was superior in the groups that were injected with secretome in comparison to the groups not treated or those transplanted with hNPCs. The characterization of the secretome revealed the presence of different players, such as brain-derived neurotrophic factor (BDNF) or pigment epithelium-derived factor (PEDF), which are known to have important functions in DAergic neuronal protection and survival [24].

Several studies have also demonstrated the effects of glial cells in the context of PD. Microglia secretome, and particularly the fraction of the medium containing molecules below $30 \mathrm{kDa}$, was shown to protect cerebellar granule neurons from 6-OHDA neurotoxicity, mainly attributed to the release of TGF$\beta 2$ [25]. The secretome of olfactory ensheathing cells (OECs), a special population of glial cells that ensheath the axons of the olfactory receptor neuron, was also shown to prevent apoptosis induced by 6-OHDA in PC12 cells [26]. This effect was mediated by the modulation of intrinsic apoptotic pathways, via up-regulation of $\mathrm{BCl}-2$, down-regulation of $\mathrm{Bax}$, and thus attenuation of mitochondrial transmembrane potential loss, which inhibited apoptosis [26].

To increase the neuroprotective effects of glial cells' secretome, other authors have modulated the expression of neurotrophic factors in the glial cells. For instance, the secretome of astrocytes overexpressing glial cell line-derived neurotrophic factor (GDNF) led to a resistance against 6-OHDA toxicity in a growing neuronal cell line (SK-N-MC) [27]. Biju and colleagues [28] have also demonstrated the amelioration of 1-metil4-fenil-1,2,3,6-tetraidropiridina (MPTP)-induced degeneration, synaptic marker staining and functional recovery after systemic administration of bone-marrow derived microglia expressing neurturin. The authors support the use of microglia as a source of sustained local delivery of neurturin, due to their ability to cross the blood brain barrier. In a recent study, OECs overexpressing nuclear receptor-related factor 1 and neurogenin 2 increased the viability of $\mathrm{PC} 12$ cells treated with the neurotoxin $\mathrm{MPP}^{+}$(1-methyl-4-phenylpyridinium), inhibited oxidative stress and apoptosis in vitro, and ameliorated the behavioral deficits in a 6-OHDA rat model cotransplanted with ventral mesencephalic cells [29].

\subsection{Mesenchymal stem cells}

MSCs are a population of adult multipotent cells with the ability to self-renew and differentiate into mesenchymal lineages, with strong immunomodulatory activities [30]. These cells can be easily isolated from different sources, including bone marrow, adipose tissue, dental pulp, and umbilical cord Wharton's Jelly (WJ), which prompt several authors to explore their use in cell transplantation strategies for PD [31-33]. The first report of MSCs' potential for PD recovery demonstrated a behavioral recovery in apomorphine-induced rotations after WJ-derived MSCs (WJ-MSCs) transplantation in the striatum of a hemiparkinsonian rat model, suggesting trophic factor secretion as a mediator of the rescue of the degenerating DAergic neurons in the substantia nigra and ventral tegmental area (VTA) [31]. Intravenous administration of rat bone marrow-derived MSCs (BM-MSCs) has also significantly ameliorated the functional deficits and preserved tyrosine hydroxylase $(\mathrm{TH})$-positive fibers in the striatum and $\mathrm{TH}$ positive neurons in the SNpc in a 6-OHDA rat model [33]. After detecting SDF-1a in the secretome of MSCs, the authors demonstrated that this chemotactic cytokine suppressed apoptotic cell death of 6-OHDA-exposed PC12 cells with consequent increase of DA release from the cells [33]. Cova and colleagues [32], have also demonstrated that human MSCs (hMSCs) transplantation in the striatum of 6-OHDA lesioned animals, five days after the toxic insult, protected the DAergic terminals and induced neurogenesis in the subventricular zone, suggesting that hMSCs in situ may provide an effective support to injured neurons through the local release of soluble factors, such as BDNF.

Despite the previous reports that establish the potential of MSCs as a therapeutic tool for PD, none of them demonstrated the acquisition of a neuronal phenotype of grafted cells, and thus put forward the secretion of neurotrophic or anti-apoptotic factors as mediators of neuroprotection. In line with this, our group showed that the secretome of mesenchymal progenitors from the human umbilical cord, besides inducing neuronal differentiation of human telencephalon neural precursor cells, it was able to stimulate the levels of proliferation, neuronal/glial survival and differentiation when injected in the rat hippocampal dentate gyrus in a similar way to the transplantation of cells [34]. Other authors explored the use of MSCs secretome (in the form of conditioned media) as a cell transplantation-free approach for PD. In vitro studies revealed the neuroprotective effect of the secretome collected from human BM-MSCs (hBM-MSCs) and human tooth germ stem cells, in 6-OHDA induced cytotoxicity of murine differentiated neural stem 
cells and SH-SY5Y cells [35,36]. Parga et al. [37] have also explored the viability of DAergic cells from different sources in response to rat BM-MSCs secretome, unveiling prostaglandin E2 receptors as main mediators of the observed neuroprotective and neurorescue activities. Additionally, our group has, also investigated the in vivo effects of hBMMSCs secretome, showing a behavioral recovery of 6-OHDA lesioned rats supported by an increase of DAergic neurons and neuronal terminals in the SNpc and striatum, respectively. Based on the proteomic analysis of the secretome, we found that BM-MSCs secreted not only important neurotrophic factors, such as vascular endothelial growth factor (VEGF), BDNF, interleukin-6 (IL-6) and GDNF, but also other potential neuroregulatory molecules, namely cystatin $C$ (Cys C), glia-derived nexin, galectin-1, and PEDF [38]. Recently, it was also demonstrated the ability of MSCs secretome to degrade extracellular a-synuclein both in vitro and in vivo, an effect partially mediated by matrix metalloproteinase- 2 (MMP-2) [39].

Another important therapeutic use of MSCs secretome for $\mathrm{PD}_{\lambda}$ is its combination with cell replacement strategies. Shintani and colleagues [40], demonstrated that pretreatment of embryonic DAergic neurons with rat BM-MSCs secretome increased their survival after grafting in a 6-OHDA rat model of PD. Yao and colleagues [41] have also reported a reduction of apomorphine-induced rotational asymmetry and improved spatial learning after transplantation of secretome-treated neural stem cells into PD rats, which was correlated with an increased cell survival and differentiation into DAergic neurons in the VTA.

Recently, the contribution of the MSCs-secreted vesicles for paracrine-mediated regeneration has also been investigated [42]. In the context of PD, Jarmalavičiūte and colleagues [43] have demonstrated that exosomes isolated from dental pulp stem cells rescued human DAergic neurons from 6-OHDA induced apoptosis. Nevertheless, this is a growing area of research, and thus more studies are required to assess the role of exosomes in the reported effects of MSCs secretome.

Other authors have modulated MSCs secretion of neurotrophic factors, either by neurotrophic exposure [44] or overexpression of a specific factor in MSCs. For instance, the transplantation of GDNF-transduced rat BM-MSCs was shown to induce protection and sprouting of DAergic terminals in the striatum of neurotoxin-induced and inflammation-induced rat PD model $[45,46]$. Human umbilical cord-MSCs transduced with hepatocyte growth factor (HGF) were also able to regenerate SH-SY5Y cells exposed to $\mathrm{MPP}^{+}$, an effect probably achieved through the regulation of intracellular $\mathrm{Ca}^{2+}$ by modulating the expression of CaBP-D28K, an intracellular calciumbinding protein [47].

\section{Modulation of cell secretome profile}

Cell plasticity is one of the most important characteristics that makes some cell populations (e.g. MSCs) a potential therapeutic tool for central nervous system (CNS) applications [48-52]. Indeed, the capacity to adapt and grow into different culture conditions indicates that MSCs could be able to change/modulate their own secretome according with the conditions in which they are cultured [53-55]. Besides the secretome modulation at the intracellular level, the modulation of external cues present in cells' microenvironment and their impact on paracrine signaling have also been explored (Figure 1). However, the mechanisms underlying this modulation by the external environment are still unknown. Harnessing this knowledge would be extremely valuable to modulate the secretome according to the context of the disease in which it would be applied. The culture conditions can be changed regarding, for instance, oxygen

\section{Extracellular Modulation Strategies}

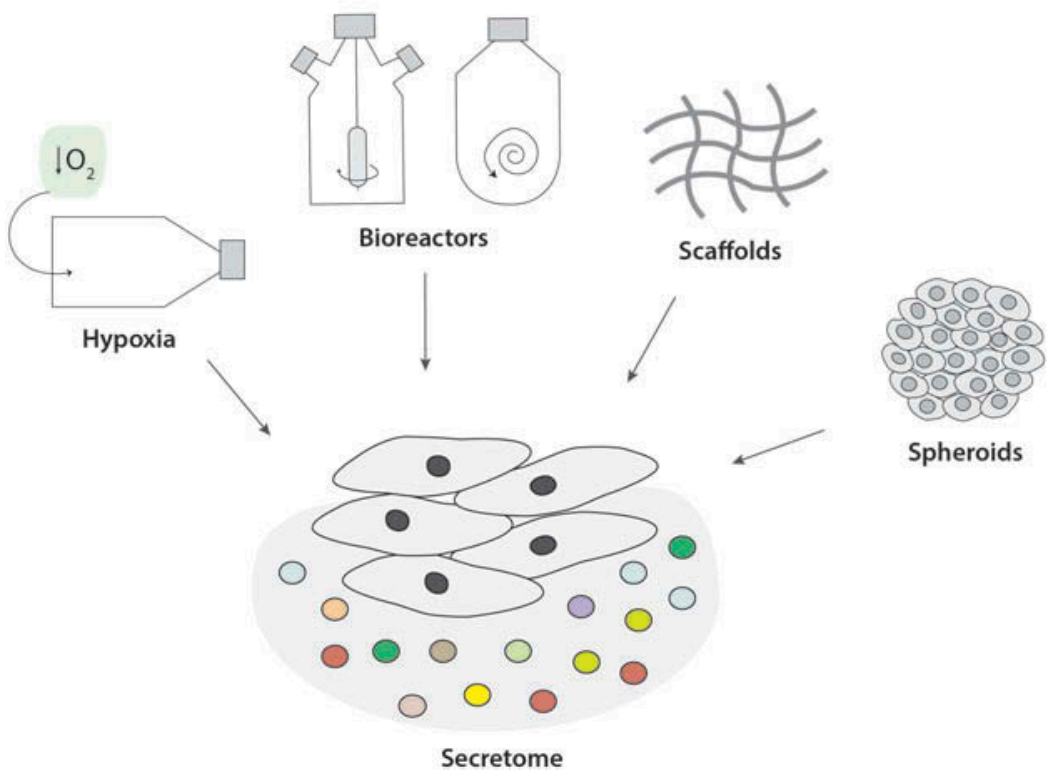

Figure 1. Strategies for the extracellular modulation of secretome from MSCs. The use of hypoxia conditions, bioreactors, hydrogels, scaffolds and spheroids has been shown to stimulate the expression of certain factors with important roles in the CNS. 
tension [56] and establishing dynamic cultures such as porous scaffolds, spheroids, or their combination, as well as encapsulation in hydrogels or even inside bioreactors [57]. Although diverse studies examined the secreted factor production of different cell types, most of recently published reports focus on MSCs due to their widespread preclinical use for tissue regeneration. Therefore, most of the concepts discussed in this topic are based on MSCs culture conditions modulation and their impact on trophic factors secretion.

\subsection{Hypoxia}

The oxygen tension present in the culture medium plays an important role in the behavior of MSCs. MSCs are usually cultured under normoxic conditions $\left(21 \% \mathrm{O}_{2}\right)$. However, in the human body, these cells are exposed to much lower concentrations of oxygen. Oxygen levels of $21 \%$ have been linked to DNA damage, leading to genomic instability and cellular senescence [58]. Several studies have been reporting that exposure to a hypoxic environment leads to changes in MSCs physiology, such as increasing the release of VEGF, impacting cellular senescence [59] cell proliferation or differentiation $[60,61]$ and consequently their regenerative potential [62].

The effects of a hypoxic environment may also influence MSCs secretome profile. On this topic, our group showed that hypoxic $\left(5 \% \mathrm{O}_{2}\right)$ and normoxic conditions-induced alterations in the secretome profile of WJ-MSCs which is translated in the capacity of both secretomes to maintain cell viability and induce differentiation of hNPCs. Furthermore, hypoxia led to the expression of 62 more proteins, when compared to normoxic culturing conditions [63]. Yuan and colleagues [64] also reported changes due to the exposure to $3 \% \mathrm{O}_{2}$ in the secretion of VEGF, nerve growth factor (NGF), BDNF, GDNF, and MMP-2. The authors suggest that hypoxia effects are mediated by the hypoxiainducible factor-1, which is important for the transcriptional activation of VEGF. In line with this, Ahmed and coworkers [65] reported that hypoxia $\left(5 \% \mathrm{O}_{2}\right)$ led to a significant increment in the expression of VEGF, BDNF and NGF. In another study, Chang and coworkers [66] administered secretome from hypoxicpreconditioned MSCs in rats with traumatic brain injury and saw that they performed significantly better in motor and cognitive function tests, had significantly less brain damage, and higher neurogenesis levels. Authors concluded that secretome had higher expression levels of VEGF and HGF.

\subsection{Bioreactors}

Cells, specifically MSCs, are usually expanded in 2D or static cultures, inside tissue culture flasks that provide a good gas exchange and not only are cost-effective, but also easy to operate. Nevertheless, if a larger number of cells are needed for clinical applications, using the mentioned systems would not be the most suitable methodology. For the scalable production of MSCs or their secretome, the development of a bioprocess that provides a controlled environment, where physiological, nutritional, chemical, and mechanical requirements are well-defined and maintained, should be mandatory [67]. Bioreactor systems can provide an interactive 3D microenvironment through the regulation of the spatial distribution of macromolecules and mechanical cues. Therefore, they have the capacity to recreate the interactions between MSCs and their microenvironment $[57,68]$.

Mechanical forces are capable of shaping MSCs' fate and bioreactors provide a tool to study the cellular response to mechanical stimulation under controlled conditions [69]. Indeed, several studies used bioreactors to successfully stimulate the differentiation of MSCs for different purposes [70-72].

MSCs grow inside the bioreactor as tissue aggregates or adherent cells to microcarriers.

Different types of bioreactors have been used for the expansion of MSCs, including stirred tank bioreactors, perfusion bioreactors, rotating well bioreactors and also microfluidic bioreactors $[57,73]$. However, few studies have addressed the use of these systems to modulate the paracrine signaling of MSCs for neurodegenerative purposes. Hupfeld and colleagues [74] studied the effects of the expansion process of MSCs derived from the amniotic membrane (AM) and from the umbilical cord (UC) on their biological characteristics. The authors concluded that the culture of MSCs in controlled bioreactor systems led to less heterogeneity between cells from different donors. These cells significantly differed from the cells cultured in flasks regarding surface markers, paracrine factors and gene expression profiles. Interestingly, VEGF was only secreted by UC-MSCs (also stimulated with cytokines) which had been expanded in bioreactors. Similarly, AM-MSCs cultivated in bioreactors secreted significantly higher levels of VEGF. Our group used computer-controlled stirred bioreactors to modulate the secretome of hBM-MSCs [75]. We showed that this system led to an enhancement of the neuroregulatory profile of hBM-MSCs secretome, which induced an increased differentiation of hNPCs. When administered in rats, even though more notorious in dynamic secretome, both secretomes induced proliferation and neuronal differentiation. All of these findings can be related with the upregulation in the dynamic secretome of important regulators/ modulators of the neurogenic and neural differentiation processes such as Cys $C$, glia-derived nexin, galactin-1, PEDF and also BDNF, VEGF, NGF, and insulin-like growth factor 1. Moreover, we identified more 28 specific molecules in the dynamic secretome which can be, at least some of them, molecules with a neuroregulatory potential.

\subsection{Culture within scaffolds or encapsulated in hydrogels}

One of the limitations of the administration of secretome per se is the impossibility of establishing a controlled release system. Indeed, we saw that when secretome was administered in a rat PD model, the effects of the secretome decreased with time, possibly due to in situ consumption [38]. In tissue engineeringbased approaches, one possible strategy to overcome this limitation is the local injection of hydrogel-embedded MSCs, enabling a long lasting secretome production with increased control over cell fate [76]. The use of biomaterials can be an essential strategy [77], because 3D culture of MSCs within scaffolds or hydrogels impacts cell physiology, enhancing endogenous extracellular matrix (ECM), and integrin expression while promoting the secretion of trophic factors [57]. However, cell 
organization and functionality are influenced by the mechanical properties of the biomaterial. Furthermore, these parameters also depend on the surface properties and spatial distribution of ECM molecules on the scaffolds and hydrogels [78]. In line with this, our group has already shown that cell adhesive cues present in 3D hydrogels regulate cell paracrine response, enhancing higher metabolic viabilities and neuronal cell densities [53]. Lee and colleagues [79] also concluded that the incorporation of carbon nanotubes within collagen hydrogels promoted the secretion of neurotrophic factors, particularly, NGF and BDNF. In another study, these neurotrophins were only expressed by MSCs isolated from gingiva cultured in a poly(lactic acid) scaffold [80].

Given that the size of the scaffolds can be restricted in static culture, they can be combined with bioreactors to provide a controllable environment for cultured cells. These dynamic systems should mimic cells' microenvironment to mobilize their full biological potential [81].

\subsection{Culture as spheroids}

Spheroids, or multicellular aggregates, are one of the simplest methods of 3D culture, which allow enhanced cell-cell interactions and better mimic the natural microenvironment of a tissue [82]. These characteristics can be the reason for the benefits obtained with this methodology. Indeed, spheroid grown MSCs showed enhanced differentiation potential [83], anti-inflammatory [84] and angiogenic properties [85], as well as improved survival after transplantation and increased paracrine expression [86]. This can be a simple approach to modulate the secretome of MSCs. Redondo-Castro and colleagues [87] showed that culture of MSCs spheroids changed the secretome of these cells leading to an enhanced secretion of cytokines involved in tissue repair and modulation of inflammation. Berg and coworkers [88] showed that spheroid cultures of adipose-derived MSC displayed higher levels of neurotrophic factors compared to adherent cultures, particularly nerve growth factor receptor and GDNF and also the integrin alpha subunit CD49b. However, the implementation of spheroid cultures demands stirring, through the use of bioreactors, or incorporation within biomaterials, to minimize spheroid agglomeration, which is the cause for cell necrosis/ apoptosis or decreased cell proliferation [82].

\section{Conclusion}

PD is an incredibly complex and multifaceted disorder, affecting millions of people worldwide. The nigrostriatal pathway, which is involved in the fine modulation of motor function is the anatomical circuit mostly affected in PD, due to the loss of DAergic neurons at the SNpc [89]. The currently available therapeutic options reflect the lack of understanding of the causes of PD, as they are designed to minimize the motor and non-motor symptomatology, by replacing the lack of DA or increasing its activity/metabolism. Nevertheless, these strategies fail at a long-term perspective as they lose their therapeutic effect over time, leading to the use of higher dosages, which has been associated with undesirable side effects [90]. Several groups have been focusing their research on disease-modifying strategies, able to prevent, slow or halt the progression of PD. The use of stem cells has emerged as a promising approach for regenerative medicine and several debilitating disorders, due to their capacity to rapidly proliferate, self-renew, and differentiate [91]. In this field, both ectodermal-origin and non-ectodermal origin stem cells have been explored in stem cell-based strategies. However, the secretome of some cell populations and their paracrine activity on other neighboring cells is now accepted as their main therapeutic action [21]. This acknowledgement together with the ethical and technical concerns associated with cell transplantation, constitute valid reasons to foment the research on the characteristics and applications of secretome.

In this review, studies that explored the potential of the secretome from neural-derived cells and MSCs in the context of PD were reported (Figure 1). Although much has been done to demonstrate the effectiveness of the secretome from different sources, a complete description of its mechanism of action is still missing. This could be justified by the lack of a clear definition about which fraction (soluble or vesicular) is mediating the evident benefits of the secretome or if they act together as a whole. Regarding the soluble fraction of secretome, the knowledge gathered until now suggests that some of its molecules, with important roles in the CNS, may act together or singly to exert their therapeutic effects (Figure 2). The characterization of the secretome of undifferentiated hNPCs through a proteomic analysis enabled the identification of several important molecules with neuroregulatory potential, namely, GDNF, BDNF, PEDF, Cys C, and also DJ-1, among others [24]. Different studies have been developed to ascertain their roles. The results indicate that all of these molecules display neuroprotective actions in the CNS through, for instance, the activation by GDNF of signaling pathways such as the mitogenactivated protein kinase (MAPK) or the protein kinase $B$, inducing the inhibition of pro-apoptotic molecules like JNK or p38 [92]. In line with this, DJ-1 modulates the levels of miR-221 in part through the activation of the MAPK/extracellular-regulated kinase pathway. miR-221 also downregulates the expression of pro-apoptotic proteins [93]. Moreover, PEDF stimulates the activation of the nuclear factor NF-KB signaling cascade, leading to the expression of BDNF and GDNF [94]. In a mice model expressing human-mutated a-synuclein, Cys $C$ upregulated VEGF and autophagy and downregulated a-synuclein and apoptosis displaying, consequently, a neuroprotective effect [95]. In another characterization study developed by our team, VEGF was also identified in the secretome of MSCs, together with some of the proteins secreted by NPCs like GDNF, BDNF, PEDF, Cys C, DJ-1, and other molecules such as IL-6 and MMP-2 [38,96]. VEGF plays a protective role in DAergic neurons either through direct mechanisms, like the activation of the neuropilin receptor expresses on DAergic neurons or indirectly by the promotion of angiogenesis, increasing vessel permeability or enhancing both glial proliferation and secretion of neurotrophic factors [97]. IL-6 may also protect DAergic neurons through signal transducers and activators of transcription pathways, leading to the increment of antioxidant enzyme activity and reactive oxygen species scavenging [98]. 


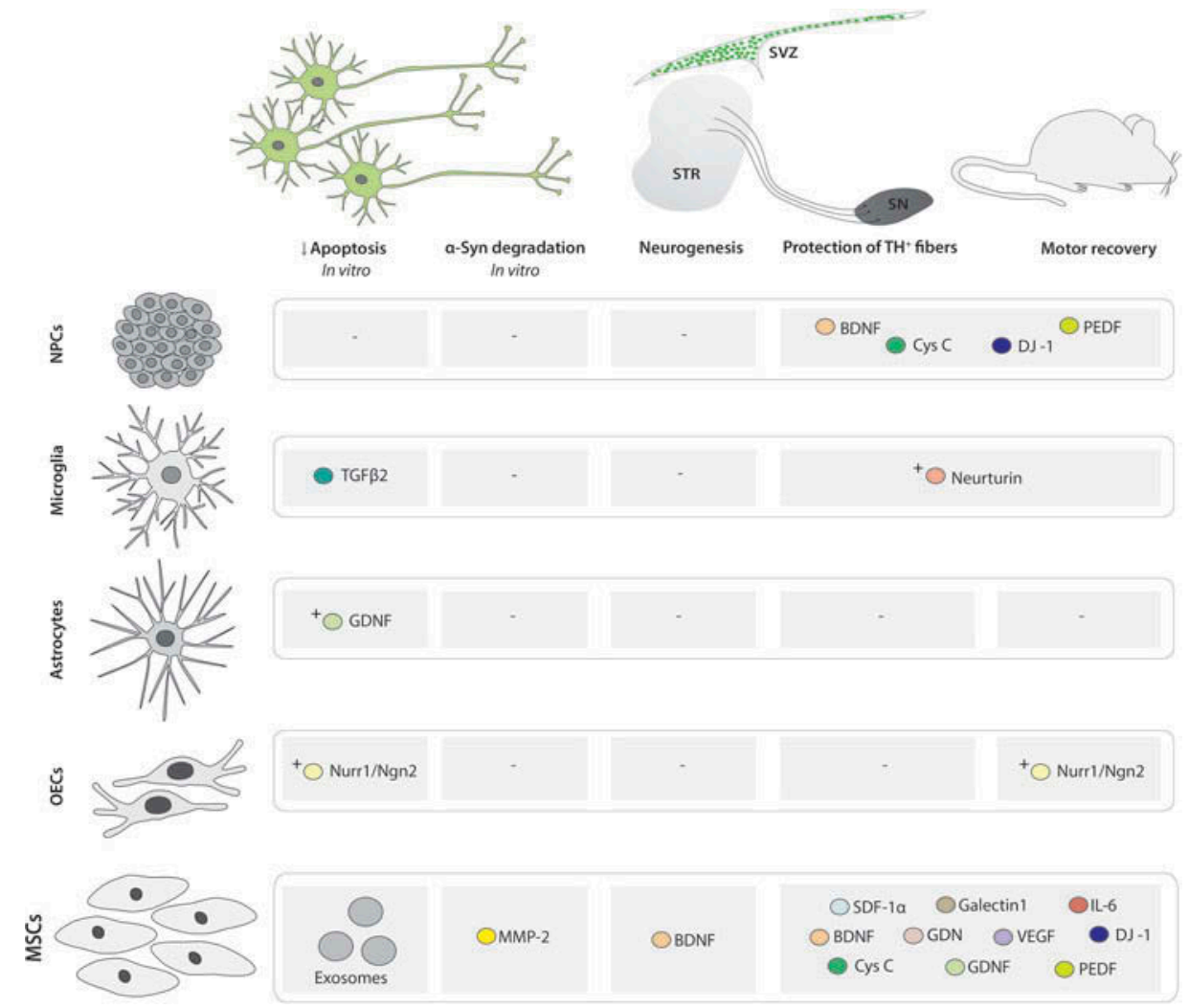

Figure 2. Overview of the impact of secretome from neural-derived cells and MSCs on different aspects relevant in the context of PD and the secreted factors that are mediating these effects. Several factors are present in cells' secretome, namely exosomes, enzymes (MMP-2), hormones, cytokines (SDF-1a, IL-6, TGF- $\beta 2$ ) and growth factors (BDNF, GDNF, VEGF, neurturin). $a$-syn = a-synuclein; BDNF = brain-derived neurotrophic factor; GDN = glia-derived nexin; GDNF $=$ glial cell linederived neurotrophic factor; IL-6 = interleukin 6; MMP-2 = matrix metalloproteinase 2; MSCs = mesenchymal stem cells; NPCs = neural progenitor cells; Nurr1/ $\mathrm{NG} 2=$ nuclear receptor-related factor 1 and neurogenin $2 ; \mathrm{OECs}=$ olfactory ensheathing cells; SDF-1 $a=$ stromal cell-derived factor $1 ;$ TGF- $\beta 2=$ transforming growth factor-beta 2; $\mathrm{TH}+=$ tyrosine hydroxylase positive; VEGF = Vascular endothelial growth factor; ${ }^{+}=$overexpression.

The presence of abnormal aggregates of a-synuclein, known as Lewy bodies, in cell bodies and processes of neurons are one of the hallmarks of PD and might result in the disruption of different cellular functions involving the mitochondria, lysosomes, endoplasmic reticulum and Golgi or also the nucleus [99]. As previously mentioned, MMP-2 may partially mediate the degradation of a-synuclein aggregates [39]. Another study showed that the secretome of BMMSCs had a neuroprotective effect in a-synuclein-enriched cellular and animal models, due to the induction of M2 microglia polarization (considered anti-inflammatory and constituted by phagocytic cells), which enhanced asynuclein clearance. The authors concluded that interleukin-4, secreted by MSCs, was the mediator of this effect [100].

MSCs have been extensively explored not only as a celltherapy strategy, but also as a source of secretome with important applications for neurodegenerative diseases and other conditions. The understanding that MSCs respond to alterations in their microenvironment, for instance, those induced by dynamic culture conditions, changing their paracrine profile, has led to the development of strategies to modulate their secretome. In this regard, recent approaches that took advantage from this feature were reviewed. Essentially, by decreasing the oxygen tension or by establishing dynamic cultures through the use of bioreactors, biomaterials or spheroid cultures, these strategies led, generally, to the upregulation of certain key molecules with important roles in the proliferation, survival, migration, and differentiation of cells in the nervous system.

The knowledge gathered until know regarding the efficacy of the secretome in animal models of PD, encourages the development of cell-free products, eliminating the need for cell transplantation strategies. Nevertheless, before the acceptance of secretome as a clinically viable option for regenerative therapies, different hurdles must be overcome. To fully harness the potential of secretome, the best strategies to efficiently modulate the secretome should be defined. Moreover, they must be easily reproducible and enable a large-scale production. Moreover, to better understand the effects and mechanism of action of secretome and translate this knowledge into clinically relevant results, more robust in vitro and in vivo models are necessary. Furthermore, other practical considerations such as mode of administration, dosage, timing and safety must also be addressed prior to clinical integration.

Cells' secretome represents a promising alternative to cellbased regenerative medicine therapies. However, beyond the great enthusiasm regarding this approach, much investigative work must be developed to build a robust and customized secretome-based therapy for PD. 


\section{Expert opinion}

Satisfactory approaches to relieve, or to slow down PD are still missing. While important gains were achieved with the current pharmacological/surgical treatments in the quality of life of PD patients, they have failed to arrest PD progression and do not promote DAergic neurons protection or differentiation. Therefore, there is an urgent need for the development of novel antiparkinsonian strategies with specific neuroprotective and neuroregenerative effects on the dopaminergic system. In recent years, new concepts mainly focused on neuroprotective/disease-modifying actions have promisingly emerged for the treatment of PD, such as the use of stem cell-based strategies. Within them, MSCs and their secretome has been presented as a promising therapeutic option. Indeed, studies have shown that the administration of secreted factors and vesicles leads to the protection of DAergic neurons and animal behavior improvement (of PD rat models), most likely due to the activation or modulation of endogenous neuro-restorative pathways. Therefore, while transdifferentiation and cell fusion remains still a (elusive) potential mechanism behind the regenerative capacity of MSCs, its secretome arises nowadays as the most likely candidate. In fact, the use of the secretome instead of cell transplantation can be more advantageous, proving to be an easily cryopreserved product, even in very small volumes, without recurrence to cryopreservation solutions, exhibiting a high stability after thawing. These characteristics make the MSCs secretome a 'ready-to-use' product, which is a very important feature when speaking about hospital routine regarding daily administration of treatments in patients.

Despite the identification of several factors within the secretome with the capacity to induce neuronal cell growth, survival and differentiation, the challenge remains regarding the full characterization of MSCs secretome. Additionally, another question that is still unanswered is, if the enthusiastic outcomes seen so far are the result of one or two factors or the result of the combination/interaction between all of the factors present in the secretome. Lastly, studies reporting improvement in animal behavior in PD animal models rely on intracranial injections to deliver the secretome. What about intraperitoneal or intravenous administrations? Would the outcome be the same?

While in animal models the secretome has proven to partially revert the phenotype of the disease, the doubt regarding its ability to reach the clinical setting remains. To approach such assumption, new protocols for the production of highquality secretome under good manufacturing practices guidelines should be developed. In this regard, bioreactors constitute an important tool to the large-scale production of secretome under controlled conditions, avoiding variability between batches and enabling the establishment of MSCs secretome as an off-the-shelf product.

Given that the number of MSCs in the human body decreases with age and that the techniques for their isolation are considered invasive, expensive, and labor-intense, research on the most suitable source of MSCs must be fostered. One possible alternative could be the use of patient-specific induced MSCs (iMSCs) derived from iPSCs.
We consider that in the coming years, research will be pursued to answer the aforementioned topics. Among them, we believe that for their importance, full characterization of the secretome and modulation strategies will receive particular attention. Indeed, these are two areas in which we are developing our work, together with the derivation of iMSCs from iPSCs.

Attending to the epidemiology and the characteristics of the disease, there is an urgent need for alternative therapeutic approaches for PD. The research on the development of cell secretome as a disease modifying strategy can have an impact on the progression of the disease by slowing it down through the protection of DAergic neurons from premature death. Furthermore, the secretome might be used as a potential addon to PD symptomatology therapies, as well as a vehicle in cell transplantation strategies to increase the survival and viability of transplanted (un)differentiated cells.

\section{Funding}

This work was supported by Portuguese Foundation for Science and Technology (FCT): Ciência 2007 Program and IF Development Grant [IF/ 00111/2013] to AJ Salgado, PhD scholarships attributed to C.R. Marques [PD/BDE/127833/2016], A. Marote [PDE/BDE/113598/2015] and B. MendesPinheiro [SFRH/BD/120124/2016] and Post-Doctoral Fellowship to F.G. Teixeira [SFRH/BPD/118408/2016]. This article has been developed under the scope of the project NORTE-01-0145-FEDER-000023, supported by the Northern Portugal Regional Operational Programme (NORTE 2020), under the Portugal 2020 Partnership Agreement, through the European Regional Development Fund (FEDER). This work has been funded by FEDER funds, through the Competitiveness Factors Operational Programme (COMPETE), and by National funds, through FCT, under the scope of the project [POCI01-0145-FEDER-007038].

\section{Declaration of interest}

The authors have no other relevant affiliations or financial involvement with any organization or entity with a financial interest in or financial conflict with the subject matter or materials discussed in the manuscript apart from those disclosed.

\section{Reviewer disclosures}

Peer reviewers on this manuscript have no relevant financial relationships or otherwise to disclose.

\section{References}

1. Pires AO, Teixeira FG, Mendes-Pinheiro B, et al. Old and new challenges in Parkinson's disease therapeutics. Prog Neurobiol. 2017;156:69-89.

2. Calabrese V, Santoro A, Monti D, et al. Aging and Parkinson's disease: inflammaging, neuroinflammation and biological remodeling as key factors in pathogenesis. Free Radic Biol Med. 2018;115:80-91.

3. Benazzouz A, Mamad O, Abedi $P$, et al. Involvement of dopamine loss in extrastriatal basal ganglia nuclei in the pathophysiology of Parkinson's disease. Front Aging Neurosci. 2014;6:87.

4. Michel PP, Hirsch EC, Hunot S. Understanding dopaminergic cell death pathways in Parkinson disease. Neuron. 2016;90:675-691.

5. LeWitt PA, Fahn S. Levodopa therapy for Parkinson disease: a look backward and forward. Neurology. 2016;86:S3-12.

6. Jimenez-Shahed J. A review of current and novel levodopa formulations for the treatment of Parkinson's disease. Ther Deliv. 2016;7:179-191. 
7. Jankovic J, Aguilar LG. Current approaches to the treatment of Parkinson's disease. Neuropsychiatr Dis Treat. 2008;4:743-757.

8. Fabbri M, Rosa MM, Abreu D, et al. Clinical pharmacology review of safinamide for the treatment of Parkinson's disease. Neurodegener Dis Manag. 2015;5:481-496.

9. Hariz G-M, Limousin P, Hamberg K. DBS means everything - for some time". Patients' perspectives on daily life with deep brain stimulation for Parkinson's disease. J Parkinsons Dis. 2016;6:335-347.

10. Parmar M, Torper O, Drouin-Ouellet J. Cell-based therapy for Parkinson's disease: a journey through decades toward the light side of the force. Eur J Neurosci. 2018;55:4763-4776.

11. Barker RA, Drouin-Ouellet J, Parmar M. Cell-based therapies for Parkinson disease-past insights and future potential. Nat Rev Neurol. 2015;11:492-503.

12. Bjorklund A, Kordower JH. Cell therapy for Parkinson's disease: what next?: cell therapy for Parkinson's disease: what next? Mov Disord. 2013;28:110-115.

13. Trounson A, McDonald C. Stem cell therapies in clinical trials: progress and challenges. Cell Stem Cell. 2015;17:11-22.

14. Lo Furno D, Mannino G, Giuffrida R. Functional role of mesenchymal stem cells in the treatment of chronic neurodegenerative diseases. J Cell Physiol. 2018;233:3982-3999.

15. Laroni A, de Rosbo NK, Uccelli A. Mesenchymal stem cells for the treatment of neurological diseases: immunoregulation beyond neuroprotection. Immunol Lett. 2015;168:183-190.

16. Isacson $\mathrm{O}$, Kordower $\mathrm{JH}$. Future of cell and gene therapies for Parkinson's disease. Ann Neurol. 2008;64:S122-S138.

17. Lindvall $O$. Clinical translation of stem cell transplantation in Parkinson's disease. J Intern Med. 2016;279:30-40.

18. Meyerrose $T$, Olson $S$, Pontow $S$, et al. Mesenchymal stem cells for the sustained in vivo delivery of bioactive factors. Adv Drug Deliv Rev. 2010;62:1167-1174.

19. Drago D, Cossetti C, Iraci N, et al. The stem cell secretome and its role in brain repair. Biochimie. 2013;95:2271-2285.

20. Salgado AJ, Sousa JC, Costa BM, et al. Mesenchymal stem cells secretome as a modulator of the neurogenic niche: basic insights and therapeutic opportunities. Front Cell Neurosci. 2015;9:249.

21. Teixeira FG, Carvalho MM, Sousa N, et al. Mesenchymal stem cells secretome: a new paradigm for central nervous system regeneration? Cell Mol Life Sci. 2013;70:3871-3882.

22. Baraniak PR, McDevitt TC. Stem cell paracrine actions and tissue regeneration. Regen Med. 2010;5:121-143.

23. Yasuhara T, Matsukawa N, Hara K, et al. Transplantation of human neural stem cells exerts neuroprotection in a rat model of Parkinson's disease. J Neurosci. 2006;26:12497-12511.

24. Mendes-Pinheiro B, Teixeira FG, Anjo SI, et al.. Secretome of undifferentiated neural progenitor cells induces histological and motor improvements in a rat model of Parkinson's disease: hNPCs secretome for Parkinson's disease repair. STEM CELLS Translational Medicine [Internet]; 2018 [cited 2018 Oct 2]. Available from: http://doi.wiley.com/10.1002/sctm.18-0009.

25. Polazzi E, Altamira LEP, Eleuteri S, et al. Neuroprotection of microglial conditioned medium on 6-hydroxydopamine-induced neuronal death: role of transforming growth factor beta-2. J Neurochem. 2009;110:545-556.

26. Feng $L$, Meng $H, W u F$, et al. Olfactory ensheathing cells conditioned medium prevented apoptosis induced by 6-OHDA in PC12 cells through modulation of intrinsic apoptotic pathways. Int J Dev Neurosci. 2008;26:323-329.

27. Safi R, Gardaneh M, Panahi Y, et al. Optimized quantities of GDNF overexpressed by engineered astrocytes are critical for protection of neuroblastoma cells against 6-OHDA toxicity. J Mol Neurosci. 2012;46:654-665.

28. Biju KC, Santacruz RA, Chen C, et al. Bone marrow-derived microglia-based neurturin delivery protects against dopaminergic neurodegeneration in a mouse model of Parkinson's disease. Neurosci Lett. 2013;535:24-29.

29. Liu Q, Qin $Q$, Sun $H$, et al. Neuroprotective effect of olfactory ensheathing cells co-transfected with Nurr1 and Ngn2 in both in vitro and in vivo models of Parkinson's disease. Life Sci. 2018;194:168-176.

30. Caplan Al, Dennis JE. Mesenchymal stem cells as trophic mediators. J Cell Biochem. 2006;98:1076-1084.

31. Weiss ML, Medicetty S, Bledsoe AR, et al. Human umbilical cord matrix stem cells: preliminary characterization and effect of transplantation in a rodent model of Parkinson's disease. Stem Cells. 2006;24:781-792.

32. Cova L, Armentero M-T, Zennaro E, et al. Multiple neurogenic and neurorescue effects of human mesenchymal stem cell after transplantation in an experimental model of Parkinson's disease. Brain Res. 2010;1311:12-27.

33. Wang $F$, Yasuhara $T$, Shingo $T$, et al. Intravenous administration of mesenchymal stem cells exerts therapeutic effects on parkinsonian model of rats: focusing on neuroprotective effects of stromal cellderived factor-1a. BMC Neurosci. 2010;11:52.

34. Teixeira FG, Carvalho MM, Neves-Carvalho A, et al. Secretome of mesenchymal progenitors from the umbilical cord acts as modulator of neural/glial proliferation and differentiation. Stem Cell Rev Rep. 2015;11:288-297.

35. Cova L, Bossolasco P, Armentero M-T, et al. Neuroprotective effects of human mesenchymal stem cells on neural cultures exposed to 6-hydroxydopamine: implications for reparative therapy in Parkinson's disease. Apoptosis. 2012;17:289-304.

36. Yalvaç ME, Yarat A, Mercan D, et al. Characterization of the secretome of human tooth germ stem cells (hTGSCs) reveals neuro-protection by fine-tuning micro-environment. Brain Behav Immun. 2013;32:122-130.

37. Parga JA, García-Garrote M, Martínez S, et al. Prostaglandin EP2 receptors mediate mesenchymal stromal cell-neuroprotective effects on dopaminergic neurons. Mol Neurobiol. 2018;55:4763-4776.

38. Teixeira FG, Carvalho MM, Panchalingam KM, et al. Impact of the secretome of human mesenchymal stem cells on brain structure and animal behavior in a rat model of Parkinson's disease. Stem Cells Transl Med. 2017;6:634-646.

39. Oh SH, Kim HN, Park HJ, et al. The cleavage effect of mesenchymal stem cell and its derived matrix metalloproteinase- 2 on extracellular a-synuclein aggregates in Parkinsonian models. Stem Cells Transl Med. 2017;6:949-961.

40. Shintani A, Nakao N, Kakishita K, et al. Protection of dopamine neurons by bone marrow stromal cells. Brain Res. 2007;1186:48-55.

41. Yao $Y$, Huang $C$, Gu $P$, et al. Combined MSC-secreted factors and neural stem cell transplantation promote functional recovery of PD Rats. Cell Transplant. 2016;25:1101-1113.

42. Marote A, Teixeira FG, Mendes-Pinheiro B, et al. MSCs-derived exosomes: cell-secreted nanovesicles with regenerative potential. Front Pharmacol. 2016;7:231.

43. Jarmalavičiūtè $A$, Tunaitis $V$, Pivoraite $U$, et al. Exosomes from dental pulp stem cells rescue human dopaminergic neurons from 6-hydroxydopamine-induced apoptosis. Cytotherapy. 2015;17:932-939.

44. Sadan O, Bahat-Stromza M, Barhum Y, et al. Protective effects of neurotrophic factor-secreting cells in a 6-OHDA rat model of Parkinson disease. Stem Cells Dev. 2009;18:1179-1190.

45. Moloney TC, Rooney GE, Barry FP, et al. Potential of rat bone marrow-derived mesenchymal stem cells as vehicles for delivery of neurotrophins to the Parkinsonian rat brain. Brain Res. 2010;1359:33-43.

46. Hoban DB, Howard L, Dowd E. GDNF-secreting mesenchymal stem cells provide localized neuroprotection in an inflammation-driven rat model of Parkinson's disease. Neuroscience. 2015;303:402-411.

47. Liu X-S, Li J-F, Wang -S-S, et al. Human umbilical cord mesenchymal stem cells infected with adenovirus expressing HGF promote regeneration of damaged neuron cells in a Parkinson's disease model. Biomed Res Int. 2014;2014:909657.

48. Kolf CM, Cho E, Tuan RS. Mesenchymal stromal cells. Biology of adult mesenchymal stem cells: regulation of niche, self-renewal and differentiation. Arthritis Res Ther. 2007;9:204.

49. Jung S, Teixeira FG, Panchalingam KM, et al. Potential therapeutic properties of human mesenchymal stem cells. J Clin Stud. 2012;4:36-40. 
50. Abdi R, Fiorina P, Adra CN, et al. Immunomodulation by mesenchymal stem cells: a potential therapeutic strategy for type 1 diabetes. Diabetes. 2008;57:1759-1767.

51. Bonfield TL, Nolan Koloze MT, Lennon DP, et al. Defining human mesenchymal stem cell efficacy in vivo. J Inflamm (Lond). 2010;7:51.

860 52. Chen Y, Shao J-Z, Xiang L-X, et al. Mesenchymal stem cells: a promising candidate in regenerative medicine. Int $\mathrm{J}$ Biochem Cell Biol. 2008;40:815-820.

53. Silva NA, Moreira J, Ribeiro-Samy $S$, et al. Modulation of bone marrow mesenchymal stem cell secretome by ECM-like hydrogels. Biochimie. 2013;95:2314-2319.

54. Oh SH, Lee SC, Kim DY, et al. Mesenchymal stem cells stabilize axonal transports for autophagic clearance of a-synuclein in Parkinsonian models. Stem Cells. 2017;35:1934-1947.

55. Jung $\mathrm{S}$, Panchalingam KM, Wuerth RD, et al. Large-scale production of human mesenchymal stem cells for clinical applications. Biotechnol Appl Biochem. 2012;59:106-120.

56. Kusuma GD, Carthew J, Lim R, et al. Effect of the microenvironment on mesenchymal stem cell paracrine signaling: opportunities to engineer the therapeutic effect. Stem Cells Dev. 2017;26:617-631.

57. Sart S, Agathos SN, Li Y, et al. Regulation of mesenchymal stem cell 3D microenvironment: from macro to microfluidic bioreactors. Biotechnol J. 2016;11:43-57.

58. Jagannathan L, Cuddapah S, Costa M. Oxidative stress under ambient and physiological oxygen tension in tissue culture. Current Pharmacol Rep. 2016;2:64-72.

59. Palumbo S, Tsai T-L, Li W-J. Macrophage migration inhibitory factor regulates AKT signaling in hypoxic culture to modulate senescence of human mesenchymal stem cells. Stem Cells Dev. 2014;23:852-865.

60. Zhang L, Yang J, Tian Y-M, et al. Beneficial effects of hypoxic preconditioning on human umbilical cord mesenchymal stem cells. Chin J Physiol. 2015;58:343-353.

61. Zhuo Y, Wang L, Ge L, et al. Hypoxic culture promotes dopaminergic-neuronal differentiation of nasal olfactory mucosa mesenchymal stem cells via upregulation of hypoxia-inducible factor-1a. Cell Transplant. 2017;26:1452-1461.

62. Liu L, Gao J, Yuan Y, et al. Hypoxia preconditioned human adipose derived mesenchymal stem cells enhance angiogenic potential via secretion of increased VEGF and bFGF. Cell Biol Int. 2013;37:551-560.

63. Teixeira FG, Panchalingam KM, Anjo SI, et al. Do hypoxia/normoxia culturing conditions change the neuroregulatory profile of Wharton Jelly mesenchymal stem cell secretome? Stem Cell Res Ther. 2015;6:133.

64. Yuan T, Zhuo Y, Su C, et al. Hypoxic and ischemic effects on gene and protein expression levels of paracrine factors by human olfactory mucosa mesenchymal-like stem cells. J Neurorestoratol. 2016;4:85-94.

65. Ahmed NE-MB, Murakami M, Kaneko S, et al. The effects of hypoxia on the stemness properties of human dental pulp stem cells (DPSCs). Sci Rep. 2016;6:35476.

66. Chang $C-P$, Chio $-C-C$, Cheong $C-U$, et al. Hypoxic preconditioning enhances the therapeutic potential of the secretome from cultured human mesenchymal stem cells in experimental traumatic brain injury. Clin Sci. 2013;124:165-176.

67. Panchalingam KM, Jung $S$, Rosenberg $L$, et al. Bioprocessing strategies for the large-scale production of human mesenchymal stem cells: a review. Stem Cell Res Ther. 2015;6:225.

68. Tandon N, Marolt D, Cimetta $E$, et al. Bioreactor engineering of stem cell environments. Biotechnol Adv. 2013;31:1020-1031.

69. Grad S, Eglin D, Alini M, et al. Physical stimulation of chondrogenic cells in vitro: a review. Clin Orthop Relat Res. 2011;469:2764-2772.

70. Cochis A, Grad S, Stoddart MJ, et al. Bioreactor mechanically guided 3D mesenchymal stem cell chondrogenesis using a biocompatible novel thermo-reversible methylcellulose-based hydrogel. Sci Rep. 2017;7:45018.

71. Agrawal P, Pramanik K, Biswas $A$, et al. In vitro cartilage construct generation from silk fibroin- chitosan porous scaffold and umbilical cord blood derived human mesenchymal stem cells in dynamic culture condition. J Biomed Mater Res A. 2018;106:397-407.

72. Stefani I, Asnaghi MA, Cooper-White JJ, et al. A double chamber rotating bioreactor for enhanced tubular tissue generation from human mesenchymal stem cells: a promising tool for vascular tissue regeneration. J Tissue Eng Regen Med. 2018;12:e42-e52.

73. King JA, Miller WM. Bioreactor development for stem cell expansion and controlled differentiation. Curr Opin Chem Biol. 2007;11:394-398.

74. Hupfeld J, Gorr IH, Schwald C, et al. Modulation of mesenchymal stromal cell characteristics by microcarrier culture in bioreactors. Biotechnol Bioeng. 2014;111:2290-2302.

75. Teixeira FG, Panchalingam KM, Assunção-Silva R, et al. Modulation of the mesenchymal stem cell secretome using computer-controlled bioreactors: impact on neuronal cell proliferation, survival and differentiation. Sci Rep. 2016;6:27791.

76. Chierchia A, Chirico N, Boeri L, et al. Secretome released from hydrogel-embedded adipose mesenchymal stem cells protects against the Parkinson's disease related toxin 6-hydroxydopamine. Eur J Pharm Biopharm. 2017;121:113-120.

77. Grandhi R, Ricks C, Shin S, et al. Extracellular matrices, artificial neural scaffolds and the promise of neural regeneration. Neural Regen Res. 2014;9:1573.

78. Chien H-W, Fu S-W, Shih A-Y, et al. Modulation of the stemness and osteogenic differentiation of human mesenchymal stem cells by controlling RGD concentrations of poly(carboxybetaine) hydrogel. Biotechnol J. 2014;9:1613-1623.

79. Lee JH, Lee J-Y, Yang SH, et al. Carbon nanotube-collagen threedimensional culture of mesenchymal stem cells promotes expression of neural phenotypes and secretion of neurotrophic factors. Acta Biomater. 2014;10:4425-4436.

80. Gugliandolo A, Diomede F, Cardelli P, et al. Transcriptomic analysis of gingival mesenchymal stem cells cultured on 3D bioprinted scaffold: a promising strategy for neuroregeneration. J Biomed Mater Res A. 2018;106:126-137.

81. Vunjak-Novakovic G, Scadden DT. Biomimetic platforms for human stem cell research. Cell Stem Cell. 2011;8:252-261.

82. Mckee C, Chaudhry GR. Advances and challenges in stem cell culture. Colloids Surf B Biointerfaces. 2017;159:62-77.

83. Yamaguchi $Y$, Ohno J, Sato A, et al. Mesenchymal stem cell spheroids exhibit enhanced in-vitro and in-vivo osteoregenerative potential. BMC Biotechnol. 2014;14:105

84. Murphy KC, Whitehead J, Falahee PC, et al. Multifactorial experimental design to optimize the anti-inflammatory and proangiogenic potential of mesenchymal stem cell spheroids. Stem Cells. 2017;35:1493-1504

85. Lee JH, Han Y-S, Lee SH. Long-duration three-dimensional spheroid culture promotes angiogenic activities of adipose-derived mesenchymal stem cells. Biomol Ther (Seoul). 2016;24:260-267.

86. Xu Y, Shi T, Xu A, et al. 3D spheroid culture enhances survival and therapeutic capacities of MSCs injected into ischemic kidney. J Cell Mol Med. 2016;20:1203-1213.

87. Redondo-Castro $\mathrm{E}$, Cunningham CJ, Miller J, et al. Changes in the secretome of tri-dimensional spheroid-cultured human mesenchymal stem cells in vitro by interleukin-1 priming. Stem Cell Res Ther. 2018;9:11.

88. Berg J, Roch M, Altschüler J, et al. Human adipose-derived mesenchymal stem cells improve motor functions and are neuroprotective in the 6-hydroxydopamine-rat model for Parkinson's disease when cultured in monolayer cultures but suppress hippocampal neurogenesis and hippocampal memory functi. Stem Cell Rev Rep. 2015;11:133-149.

89. Gopalakrishna A, Alexander SA. Understanding Parkinson disease. J Neurosci Nurs. 2015;47:320-326.

90. Cacabelos R. Parkinson's disease: from pathogenesis to pharmacogenomics. Int J Mol Sci. 2017;18:551.

91. Shafiq M, Jung Y, Kim SH. Insight on stem cell preconditioning and instructive biomaterials to enhance cell adhesion, retention, and engraftment for tissue repair. Biomaterials. 2016;90:85-115.

92. Du Y, Li X, Yang D, et al. Multiple molecular pathways are involved in the neuroprotection of GDNF against proteasome inhibitor 
induced dopamine neuron degeneration in vivo. Exp Biol Med. 2008;233:881-890.

93. Oh SE, Park H-J, He L, et al. The Parkinson's disease gene product DJ-1 modulates miR-221 to promote neuronal survival against oxidative stress. Redox Biol. 2018;19:62-73.

94. Falk T, Gonzalez RT, Sherman SJ. The Yin and Yang of VEGF and PEDF: multifaceted neurotrophic factors and their potential in the treatment of Parkinson's disease. Int J Mol Sci. 2010;11:2875-2900.

95. Zou J, Chen Z, Wei X, et al. Cystatin C as a potential therapeutic mediator against Parkinson's disease via VEGF-induced angiogenesis and enhanced neuronal autophagy in neurovascular units. Cell Death Dis. 2017;8:e2854.

96. Pires AO, Mendes-Pinheiro B, Teixeira FG, et al. Unveiling the differences of secretome of human bone marrow mesenchymal stem cells, adipose tissue-derived stem cells, and human umbilical cord perivascular cells: a proteomic analysis. Stem Cells Dev. 2016:25:1073-1083.

97. Yasuhara T, Shingo T, Kobayashi K, et al. Neuroprotective effects of vascular endothelial growth factor (VEGF) upon dopaminergic neurons in a rat model of Parkinson's disease. Eur J Neurosci. 2004;19:1494-1504.

98. Hirano T, Ishihara K, Hibi M. Roles of STAT3 in mediating the cell growth, differentiation and survival signals relayed through the IL-6 family of cytokine receptors. Oncogene. 2000;19:2548-2556.

99. Wong YC, Krainc D. a-synuclein toxicity in neurodegeneration: mechanism and therapeutic strategies. Nat Med. 2017;23:1-13.

100. Park HJ, Oh SH, Kim HN, et al. Mesenchymal stem cells enhance a-synuclein clearance via M2 microglia polarization in experimental and human parkinsonian disorder. Acta Neuropathol. 2016;132:685-701 